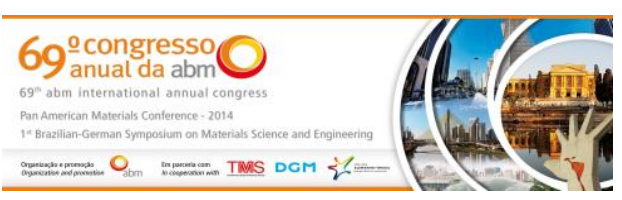

Tema: Fundição

\title{
CARACTERIZACIÓN MICROESTRUCTURAL DE UN MATERIAL BASE COBRE PARA FABRICAR MOLDES PARA LA INDUSTRIA DEL PLÁSTICO*
}

\section{Resumen}

Ernesto Gustavo Maffia ${ }^{1}$ Alfredo González ${ }^{2}$

Flavio Marchiori ${ }^{3}$

En la actualidad, el moldeo por inyección es una tecnología muy utilizada en el procesamiento del plástico pues permite fabricar formas geométricas complejas. Para fabricar los moldes se utiliza ampliamente la aleación C17200 (Cu-2\%Be), pero están en desarrollo algunas alternativas con aleaciones base cobre libres de berilio, que tienen valores de dureza por encima de los 30 Rockwell $\mathrm{C}$ y buena conductividad. Las aleaciones base de cobre, tipo CuNiSiCr son las más adecuadas como reemplazo. El siguiente trabajo trata del estudio y desarrollo del proceso de fabricación, por fusión, colado y conformado, de una aleación tipo CuNiSiCr de alta dureza y buena conductividad para ser utilizada en la fabricación de moldes de inyección para la industria del plástico. Los resultados preliminares de esta investigación demuestran que a través del aumento sistemático en el contenido de níquel y silicio se consigue un aumento de la dureza ( $>32$ Rockwell C) y una aceptable conductividad eléctrica ( $\approx 25 \% \mid A C S$ ). El fenómeno segregación interfiere fuertemente en los tratamientos termomecánicos, pues se forma una segunda fase interdendrítica, de características frágiles, que genera fracturas en el conformado. Los resultados demuestran que la aplicación del recocido total y control de la temperatura durante la forja minimizan este problema.

Palabras-clave: Material para matrices y moldes de inyección; Aleación base cobre; Microestructuras.

\section{MICROESTRUCTURAL CHARACTERIZATION FROM A COPPER BASE ALLOY TO MANUFACTURE MOLDS IN PLASTIC INDUSTRY}

\begin{abstract}
At present, injection moulding is widely used in plastic processing because it allows production of complex shapes. To produce moulds C17200 (coppeer-berylium) alloy is widely used, but few beryllium free copper base alternatives and with hardness values above 30 Rockwell C and good conductivity alloys are in development. Copper base alloys, CuNiSiCr type, are most suitable as a replacement. The following work deals with the study and development of the manufacturing process through melting, casting and forming from a high strength and good conductive CuNiSiCr type alloy to be used in the manufacture of injection moulds in the plastics industry. Preliminary results of this research show that through a systematic increase in nickel and silicon content an increase in hardness $(>32$ Rockwell C) and an acceptable electrical conductivity (> 27\% IACS) is achieved. The segregation phenomenon interferes strongly in thermo mechanical treatments, because a second interdendritic phase with fragile characteristics is generated provoking fractures when forming. The results demonstrate that application of the full annealing and temperature control during forging minimize this problem

Keywords: Material dies and injection molds; Copper alloy base; Microstructures.

1 Dr. Ing., Laboratorio de Investigaciones en Metalurgia Física (LIMF), Departamento de Mecánica, Facultad de Ingeniería, Universidad Nacional de La Plata (UNLP), BA, Argentina; ernesto.maffia@ing.unlp.edu.ar.

2 Dr. Ing., Laboratorio de Investigaciones en Metalurgia Física (LIMF), Departamento de Mecánica, Facultad de Ingeniería, Universidad Nacional de La Plata (UNLP), BA, Argentina.

3 Sócio Gerente de Coopersol SA, Lanus, BA, Argentina.
\end{abstract}

* Contribuição técnica ao 69ํ Congresso Anual da ABM - Internacional e ao 14º ENEMET - Encontro Nacional de Estudantes de Engenharia Metalúrgica, de Materiais e de Minas, 21 a 25 de julho de 2014, São Paulo, SP, Brasil. 


\section{INTRODUCCIÓN}

En la actualidad, el moldeo por inyección es una tecnología del procesamiento de plástico muy utilizada para fabricar componentes con formas geométricas complejas y con altos volúmenes de producción. Para lograr esta meta, es indispensable un molde de buenas cualidades mecánicas y metalúrgicas, con una elaboración muy precisa, y duración aceptable.

En muchos casos se utiliza aceros especiales para fabricar los moldes pues estos cumplen con las exigentes condiciones de trabajo. Sin embargo, no son los únicos materiales disponibles hoy día para fabricar moldes y matrices. Existe una gran gama de materiales, entre los cuales podemos encontrar, además de los aceros, materiales de colada, materiales no metálicos y materiales cerámicos.

Como la tarea principal del molde de inyección es recibir y distribuir el material plástico fundido, posteriormente enfriar y expulsar la pieza plástica moldeada, conviene tener en cuenta algunas consideraciones en cuanto a las propiedades que deben reunir los materiales a utilizar.

Estos materiales deben contar con:

- Buena mecanizabilidad, poder ser troquelados en frío y poder ser tratados térmicamente.

- Resistencia a la compresión, a la temperatura y a la abrasión

- Aptitud para el pulido

- Tener deformación reducida

- Buena conductividad térmica

- Buena resistencia Química

- Tratamiento térmico sencillo.

Dentro de las aleaciones no ferrosas disponibles, la aleación base cobre C17200 (Cu-2\%Be) es una de las mas utilizadas por su alta resistencia y por su alta conductividad térmica. Se utiliza ampliamente para moldes de inyección o bien como núcleos o insertos en acero para moldes. La conductividad térmica de la aleación base cobre C17200 es de 3 a 4 veces mayor que la de cualquier acero, y esta propiedad asegura una rápida y uniforme eliminación del calor, minimizando la distorsión de la pieza, las deformaciones del molde, una pobre reproducción de los detalles del producto. En muchos casos también puede reducir de forma significativa los tiempos de ciclo.

A pesar de las óptimas propiedades de las aleaciones $\mathrm{Cu}-\mathrm{Be}$, surge la necesidad de aleaciones libres de berilio, que presenten valores de dureza por encima de los 30 Rockwell C, manteniendo al mismo tiempo una buena conductividad. Una posible alternativa a este material es una aleación de cobre-níquel-silicio-cromo, forjada o fundida con alta dureza [1-3]. En general, la aleación debería componerse de $8 \%$ a $12 \%$ de níquel, 1 a $3.5 \%$ silicio, $0,5 \%$ a $2,0 \%$ de cromo, y el resto cobre. Con esta composición química y técnicas precisas de conformado, se consigue una dureza por encima de 30 Rockwell $\mathrm{C}$, junto con una conductividad eléctrica por encima de $24 \%$ del cobre puro, a través de un tratamiento térmico.

Esta aleación puede ser utilizada, tanto como un material para matrices de moldeo, para extrusión de piezas de plástico o para cualquier pieza que requiera como propiedad esencial en servicio, un alto modulo elástico, como en el caso de los resortes de contacto eléctrico de equipos de computación y/o equipos eléctricos.

En el caso de ser utilizada como material para matrices, el incremento en la dureza permite a la material soportar las altas presiones de cierre del molde, sin distorsion. Esta dureza también permite resistir la erosión del material plástico fundido, en

* Contribuição técnica ao 69ํ Congresso Anual da ABM - Internacional e ao 14º ENEMET - Encontro Nacional de Estudantes de Engenharia Metalúrgica, de Materiais e de Minas, 21 a 25 de julho de 2014, São Paulo, SP, Brasil. 


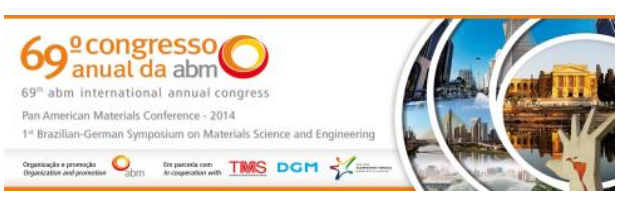

particular cuando contiene en su interior material fibroso. Además, como es una aleación con una excelente difusividad térmica, permite absorber el calor y reducir el tiempo de enfriamiento del molde, disminuyendo de ese modo el tiempo de ciclo para las operaciones de conformado. En el caso de ser usado como de resortes de contacto, este material debe permitir ser fácilmente conformado en alguna condición blanda y que a continuación, endurecerlo por medio un simple tratamiento térmico parar proporcionar las propiedades elásticas necesarias y ser rápidamente puesto en servicio.

Las propiedades con las que cuenta la aleación $\mathrm{CuNiSiCr}$ hacen de ésta, un material adecuado para una amplia variedad de aplicaciones, pero especialmente donde se requiera una combinación de alta conductividad térmica, resistencia a la corrosión y buena terminación superficial.

El objetivo de este desarrollo es caracterizar por técnicas de microscopia y dureza el proceso de fusión y tratamientos termomecánicos de la aleación CuNiSiCr a utilizarse en la confección de moldes para la industria del plástico.

\section{MATERIALES Y MÉTODOS}

Para la fabricación de la aleación CuNiSiCr se parte de elementos puros para evitar la contaminación del material final y tener la certeza que la conductividad no es afectada por contaminantes. El desarrollo de la fusión se realiza aplicando procedimientos estándar de fundición, utilizando un horno de fosa, calefaccionado con gas natural, con un crisol de grafito estándar y bajo atmosfera de protección. En esta etapa es importante garantizar las condiciones del tiempo de permanencia a las temperaturas adecuadas para lograr la disolución del silicio y el cromo.

La secuencia de fusión es la siguiente:

(a) El cobre y el níquel se funden en el horno de fosa al máximo régimen de funcionamiento, bajo cobertura de carbón mineral;

(b) Cuando cobre y el níquel están en estado liquido, se añade el fósforo (como aleación madre de cobre-15\% fósforo), para desoxidar la masa liquida.

(c) a posteriori se añaden las cantidades requeridas silicio y de cromo, como aleaciones madre, para producir la composición deseada;

(d) La masa fundida se cuela tan pronto como se completa la solución del silicio y de los demás aleantes en la masa liquida.

Los lingotes se obtienen por colado por gravedad en coquilla, de diámetro $150 \mathrm{~mm}$ y $600 \mathrm{~mm}$ de altura, utilizando como método de colada el sistema el denominado "Durville". Una vez obtenido el lingote, este se analiza químicamente para determinar la composición de la aleación: $11 \% \mathrm{Ni}, 1.5 \% \mathrm{Cr}$, y $2.5 \%$ de Si. Posteriormente, se forjan varias probetas a temperaturas entre 790 a $900^{\circ} \mathrm{C}$ llevando el material a una sección transversal adecuada, no menor de $12 \mathrm{~mm}$. En base a las muestras obtenidas, se realiza un solubilizado, temple y un posterior envejecido. Todas las etapas de fabricación se caracterizan a través de Microscopía Óptica (M.O.) con un microscopio óptico (Leica) y Microscopia Electrónica de Barrido (M.E.B.) con Microanálisis Químico por Espectroscopia de Rayos X por Dispersión en la Energía (E.D.S.). Las muestras caracterizadas se preparan con las técnicas metalográficas convencionales de desbaste, pulido, y ataque, utilizando cloruro férrico y dicromato de potasio para revelar la microestructura. Por último se emplea un durómetro marca FRANK modelo 532, con penetrador certificado (OSHMA). El método utilizado es Brinell empleando una bolilla de acero de $2,5 \mathrm{~mm}$ de diámetro y una carga de 187.5

\footnotetext{
* Contribuição técnica ao 69ํㅡㄴ Congresso Anual da ABM - Internacional e ao 14 ENEMET - Encontro Nacional de Estudantes de Engenharia Metalúrgica, de Materiais e de Minas, 21 a 25 de julho de 2014, São Paulo, SP, Brasil.
} 


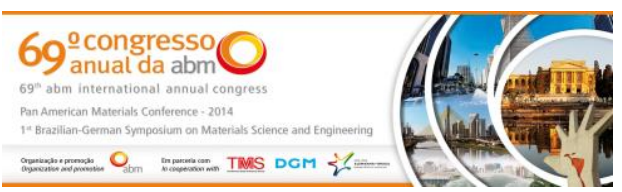

$\mathrm{kg}$, la Norma de referencia empleada es ASTM 10 y los valores obtenidos son indicados en la tabla 1.

Para evaluar los resultados de este desarrollo a nivel industrial se caracteriza la microestructura en cada una de las etapas de fabricación. Las etapas a evaluar son: a) etapa de colado y solidificación del lingote, b) tratamiento térmico de homogeneizado, c) forja, d) tratamiento térmico de endurecimiento por precipitación, e) relevamiento de dureza y conductividad

\section{RESULTADOS}

\section{1caracterizacion del Material De Colada}

Las observaciones iniciales revelan una microestructura de colada altamente segregada, con una significativa cantidad de segunda fase en borde de grano, como se observa en la figura 1, siendo el tamaño del grano de, aproximadamente $40 \mu \mathrm{m} \pm 10$, sin observarse porosidad o rechupes en las demás macros.

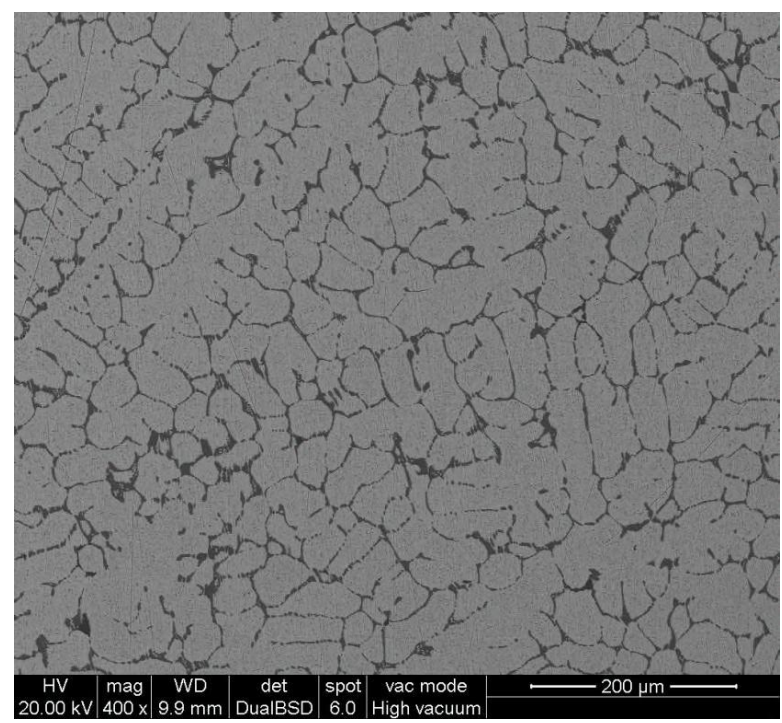

Figura 1- microestructura dendrítica de la aleación CuNiSiCr al salir de la colada.

En el desmolde se producen roturas y es necesario conocer su origen. En la figura 2 puede observarse una micrografía mostrando un superficie de fractura tipo frágil, intergranular o de aspecto rocoso producida en el desmolde y temple del lingote colado.

\footnotetext{
* Contribuição técnica ao $69^{\circ}$ Congresso Anual da ABM - Internacional e ao 14ํㅡㄹ ENEMET - Encontro Nacional de Estudantes de Engenharia Metalúrgica, de Materiais e de Minas, 21 a 25 de julho de 2014, São Paulo, SP, Brasil.
} 

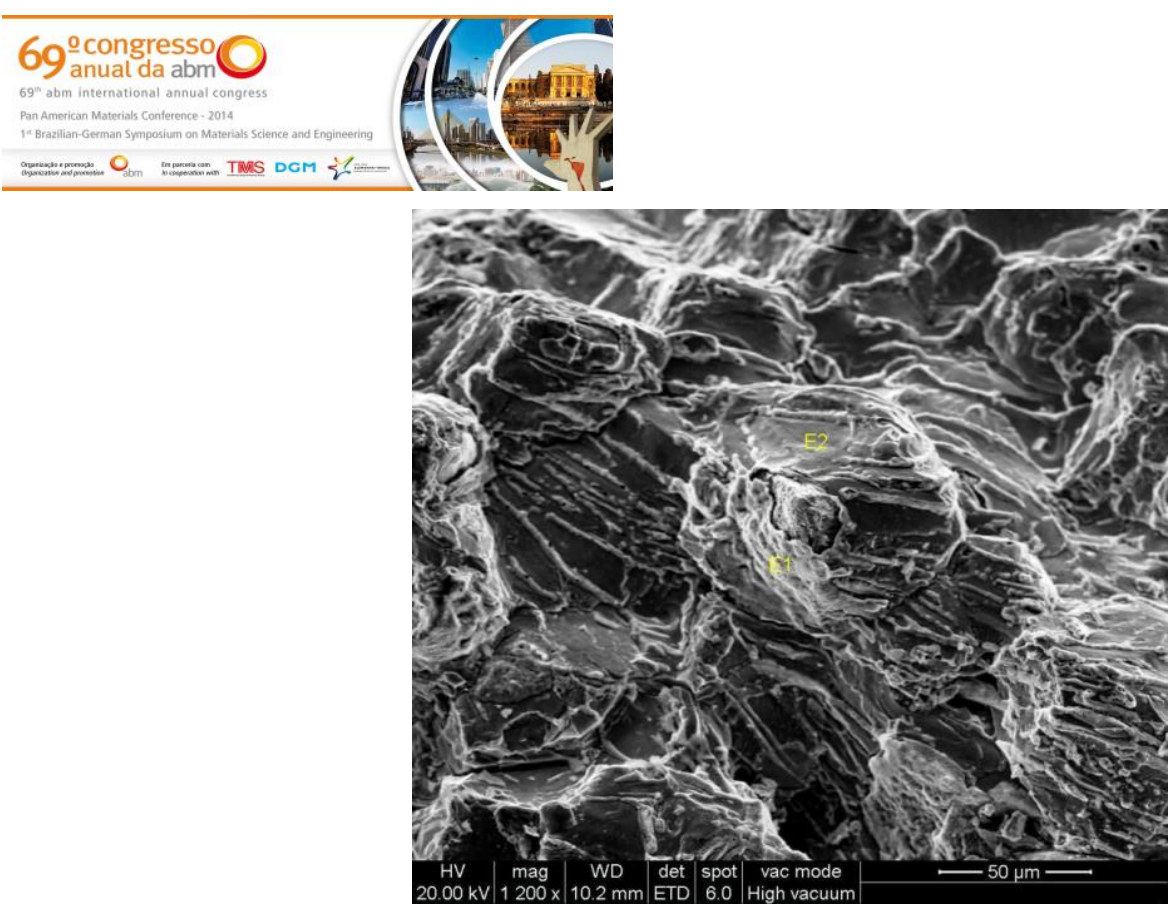

Figura 2- Superficie de fractura de la aleación CuNiSiCr al salir del temple y estructura dendrítica [4].

Los análisis vía EDS de las fases expuestas en las superficies de fractura se presentan en la tabla 1 . Se observa que estas fases presentan al silicio, níquel y cromo segregado y en modo continuo en los contornos de grano, como también se observa en la figura 1.

\begin{tabular}{|c|l|l|l|l|}
\hline \multicolumn{5}{|c|}{ Tabla 1- Elementos encontrados en el análisis vía EDS } \\
\hline Zona examinada & Silicio & Níquel & Cromo & Cobre \\
\hline E1 & Si & si & si & resto \\
\hline E2 & trazas & trazas & trazas & En su mayoría \\
\hline
\end{tabular}

\subsection{Tratamiento Térmico de Homogeneizado}

Los primeros intentos de forjar la aleación CuNiSiCr en condición as cast, demuestran una gran dificultad, con roturas multiples. Evidentemente, la estructura segregada del material dificulta el conformado. De modo que es necesario realizar un homogenizado para disminuir la segregación [5].

En la siguiente secuencia de micrografías muestra la estructura resultante del tratamiento térmico de homogeneizado de un lingote de la aleación CuNiSiCr a $900^{\circ} \mathrm{C}$ por $7,5 \mathrm{~h}$ y templadas en agua

\footnotetext{
* Contribuição técnica ao 69 Congresso Anual da ABM - Internacional e ao 14ํㅡㄹ ENEMET - Encontro Nacional de Estudantes de Engenharia Metalúrgica, de Materiais e de Minas, 21 a 25 de julho de 2014, São Paulo, SP, Brasil.
} 

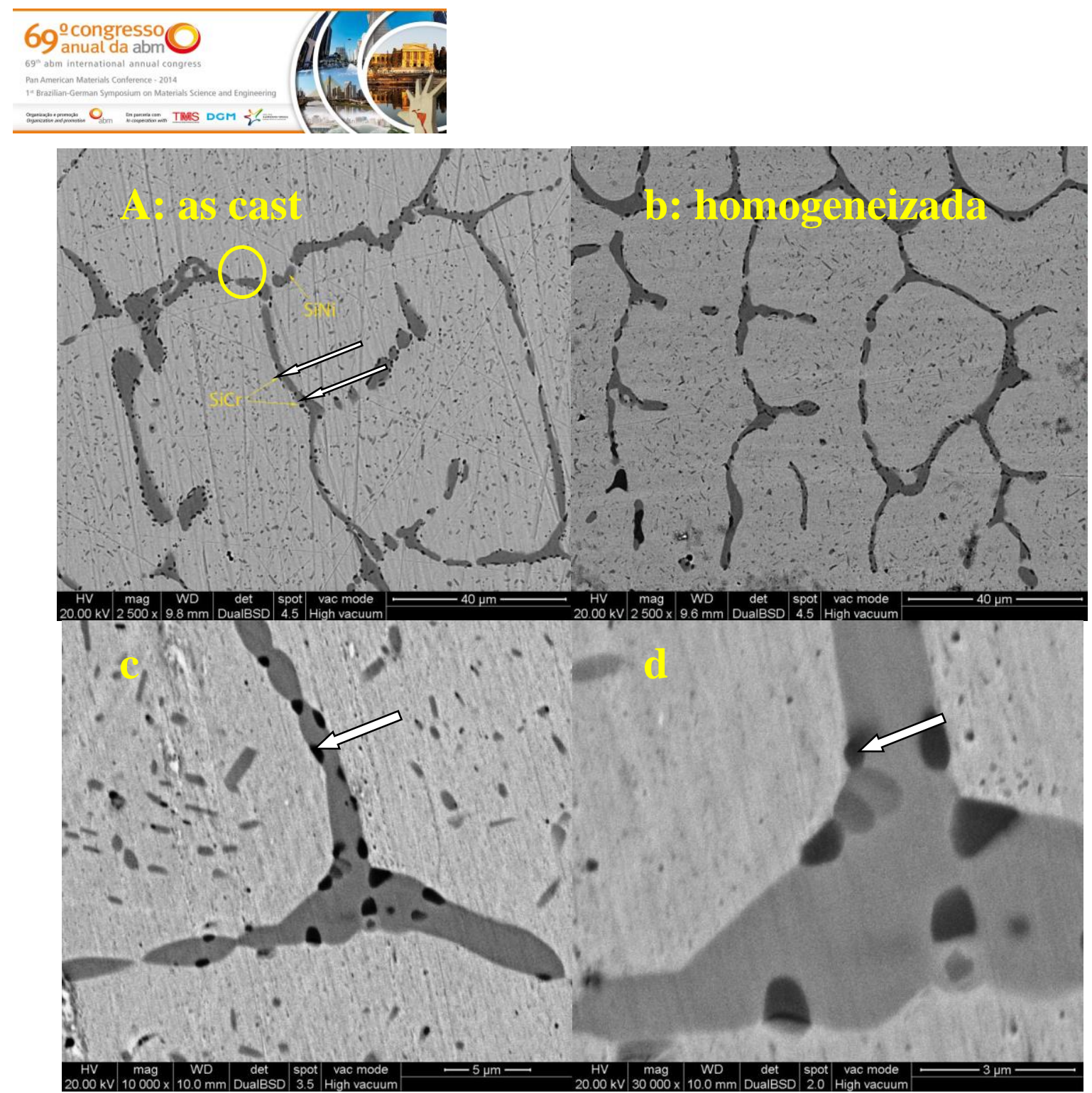

Figura 3- Aleación CuNiSiCr. (a) as cast. (b) homogeneizada y templadas en agua a temperatura ambiente. (c) y (d) ampliaciones de las regiones con segundas fases.

En cuanto a la morfología, tipo y cantidad de segundas fases formadas durante la solidificación, la secuencia de ampliaciones de la microestructura presentadas en las figuras (c) y (d) permiten ubicar y distinguir claramente la morfología de estas fases.

Los análisis vía EDS de las zonas grises (circulo), permiten confirmar que son siliciuros de níquel y se ubican rodeando los granos de cobre. Dentro de esta fase se observa precipitación de otra fase de color negro, que resulta ser un siliciuro de cromo (flecha). Según la literatura, estas dos fases precipitan simultáneamente en el último líquido que solidifica. Sin embargo, también se encuentran dentro de los granos, conformando un modo de precipitación discontinua. Las micrografías no muestran efecto alguno en lo referente a la disminución de la segregación con el homogeneizado, por lo que, este tratamiento no debería ser parte del proceso de fabricación, pues no puede disminuir la segregación.

\subsection{Tratamiento Térmico de Recocido Completo}

Como el homogeneizado es un procedimiento que no produce la disminución de la segregación, es necesario entonces producir otro tipo de distribución de precipitados en la microestructura a conformar.

Se utiliza el recocido total con el fin de aumentar la trabajabilidad del material [5]. 


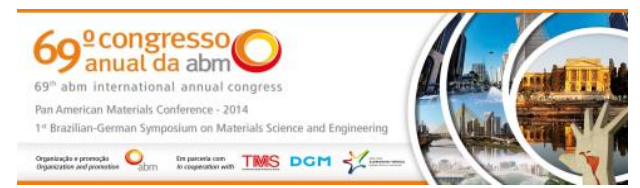

A seguir se presentas micrografías de los resultados del recocido total realizado a $900^{\circ} \mathrm{C}$ por espacio de $7,5 \mathrm{hs}$ y luego enfriadas en horno por $24 \mathrm{Hs}$ hasta temperatura ambiente. En la figura 4 se presentan los resultados.

Después del recocido total se observa un aumento en la cantidad de precipitados dentro de los granos. La morfología de estos puede apreciarse en la micrografía (b) de la figura 4. Estos precipitados presentan contornos poligonales y además hay precipitados de siliciuro de cromo pegados a los siliciuros de níquel, de manera similar a los eventos que ocurren en los límites de granos. Las cercanías de las fronteras de los granos se presentan libres de precipitación.

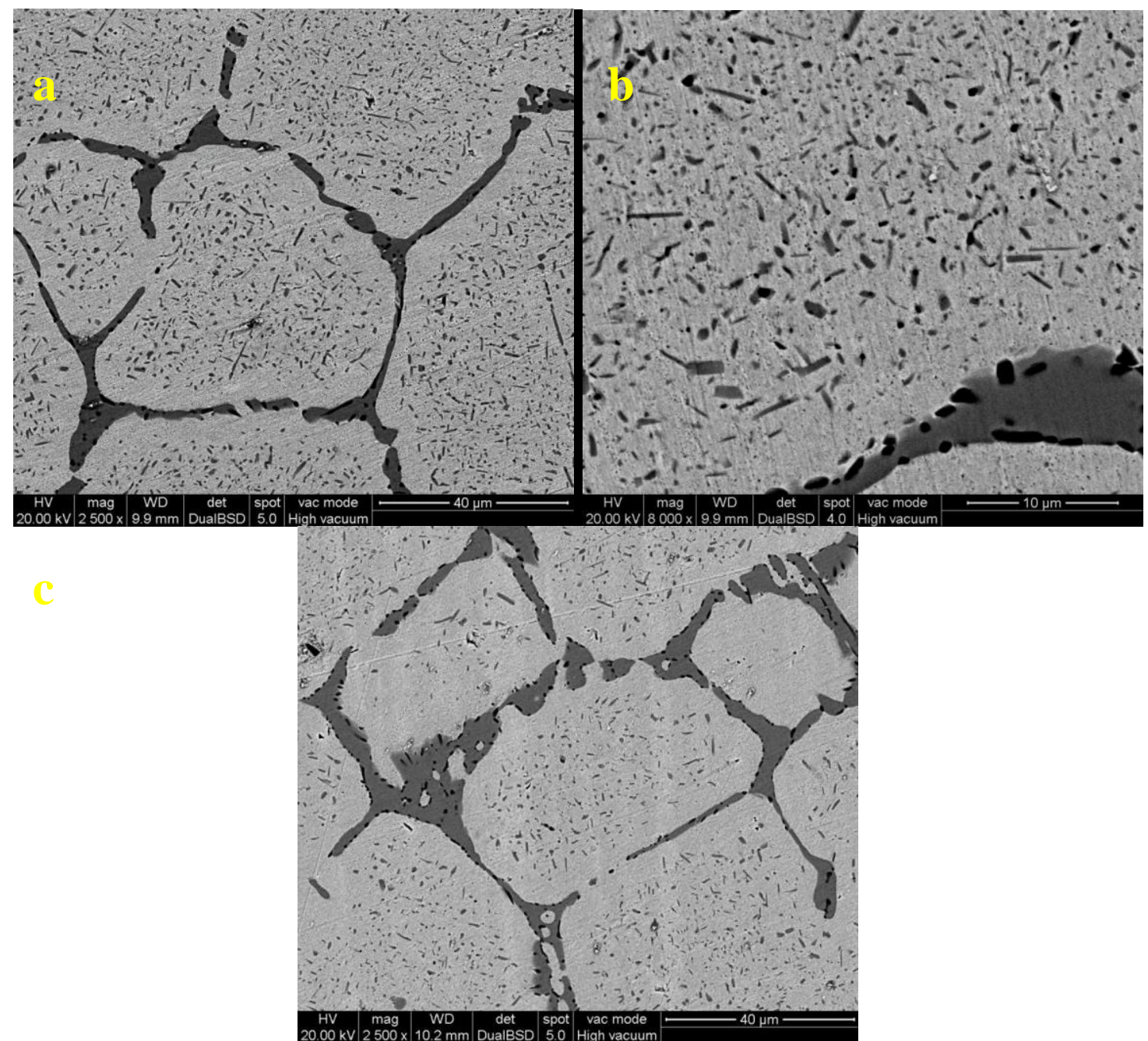

Figura 4- Diferentes modos de precipitación: (a y b) con recocido total, (c) templada.

\section{DISCUSIÓN}

En la fabricación industrial de aleaciones base cobre, una de las formas habituales de disminuir los tiempos de fabricación es desmoldar y enfriar en agua los lingotes, aun calientes y a altas temperaturas. Este modo de enfriamiento no genera fisuras ni distorsiones en la mayoría de las aleaciones base cobre termo tratables por tener estas una matriz dúctil que absorbe las tensiones/distorsiones durante el violento enfriamiento. Sin embargo, cuando la aleación Cu.11\%Ni-1,5\%Cr-2,5\%Si se desmolda y se la templa desde altas temperaturas, muestra una gran fragilidad, manifestándose en forma de grandes fisuras que inutilizan el lingote. La observación de la morfología de la fisura muestra signos evidentes de rotura intergranular. * Contribuição técnica ao 69 Congresso Anual da ABM - Internacional e ao 14ํㅡㄹ ENEMET - Encontro Nacional de Estudantes de Engenharia Metalúrgica, de Materiais e de Minas, 21 a 25 de julho de 2014, São Paulo, SP, Brasil. 
Evidentemente los límites de grano son frágiles y esto puede estar ocurriendo por fusión incipiente de las fases en borde de grano o estas fases pueden no absorber las diferentes tensiones que desarrollan durante el enfriamiento, llegándose a la rotura intergranular para aliviar las tensiones. Este hecho permite comprobar que la fase en límite de grano es frágil a las elevadas temperaturas de desmolde, lo cual obliga a enfriamientos lentos en el molde.

Según W.W. Edens et al, las fases formadas durante la solidificación son siliciuros de níquel y de cromo (figura 3) continuas e incoherentes a lo largo de los limites de granos. Generalmente, tienen un leve efecto de endurecimiento sobre la aleación pero un gran efecto en las operaciones de conformación.

En este tipo de aleaciones, estas fases precipitan simultáneamente en el último líquido que solidifica en los bordes de grano y también dentro de los granos, en forma discontinua, indicando que están solidas en la solución liquida.

Como el material debe ser conformado para las diferentes aplicaciones industriales, debería estar en la condición de máxima ductilidad al momento de llegar a la forja o a la laminadora. Esta ductilidad máxima no se logra con el tratamiento de homogeneizado, el cual resulta ineficiente para eliminar o disminuir de la segregación.

De modo que, el recocido total es mas adecuado para lograr una morfología de precipitados incoherentes dentro de los granos, en una matriz con escasos elementos disueltos en solución. La morfología de estos puede apreciarse en la micrografía (b) de la figura 4, la cual es poligonal. Además, las adyacencias de los granos se presentan libres de precipitación. Al someter varias probetas al proceso de forja libre, el cual es un método extremo para verificar la trabajabilidad de este tipo de microestructura, se encuentran óptimos resultados. La caracterización de estas estructuras muestra que las segundas fases están quebradas y diseminadas por toda la matriz.

Este tipo de distribución de precipitados se acerca a una situación ideal en cuanto a la optimización de propiedades: una matriz dúctil y saturada en elementos para precipitar en el envejecido final y fases incoherentes, grandes y dispersas en la matriz que no aumentan el endurecimiento por trabajado y por ende, no aumentan la resistencia durante el conformado.

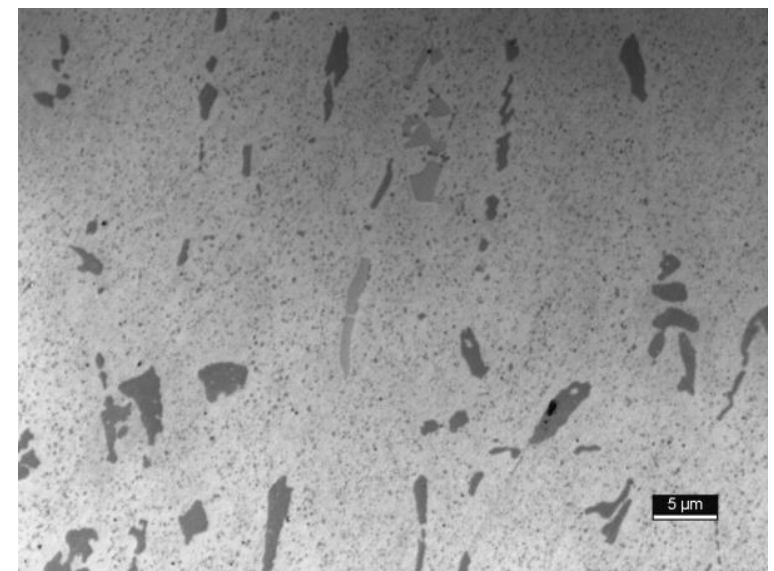

Figura 5- Aleación CuNiSiCr con recocido total y forjada.

Después de la forja, la muestra es envejecida a temperaturas de $510^{\circ} \mathrm{C}$. Posteriormente, se realiza un barrido de dureza en la placa forjada y tratada térmicamente, obteniéndose durezas de 32HRC, aproximadamente, con una 


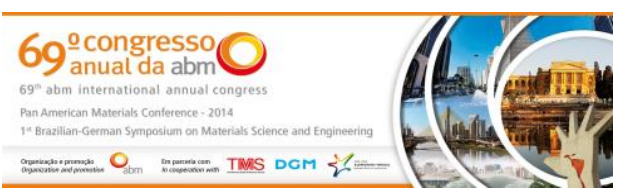

conductividad de alrededor del $27 \%$ IACS. Estas características son comparables con otras aleaciones para moldes, como se presenta en la siguiente tabla:

Tabla 2 - comparación de propiedades útiles en la selección de materiales para moldes de inyección de plásticos

\begin{tabular}{|c|c|c|}
\hline & $\begin{array}{c}\text { Conductividad térmica }[\mathrm{W} / \mathrm{m} . \mathrm{k}], \\
\left(20-25^{\circ} \mathrm{C}\right)\end{array}$ & Dureza [HRC] \\
\hline $\begin{array}{c}\text { Acero } \\
\text { W1.2738 }\end{array}$ & 34 & $44-52$ \\
\hline$H-13$ & 26 & $30-32$ \\
\hline$P-20$ & 41 & $39-45$ \\
\hline C17200 & $105-130$ & $\approx 32$ \\
\hline CuNiSiCr & $105-115$ & (media de 10 medidas) \\
\hline
\end{tabular}

\section{CONCLUSIONES}

La caracterización por técnicas de microscopia, dureza y conductividad del proceso de fabricación de la aleación CuNiSiCr a utilizarse en la confección de moldes para la industria del plástico, demuestra que este material puede ser elaborado con éxito si se aplica una adecuada combinación de recocido total, forja y control de temperaturas, próximas a los $900^{\circ} \mathrm{C}$. El proceso total promueve la destrucción total de la estructura de colada segregada y genera un material trabajable por forja.

Se concluye que es posible su fabricar una aleación $\mathrm{Cu} .11 \% \mathrm{Ni}-1,5 \% \mathrm{Cr}-2,5 \% \mathrm{Si}$, con valores de conductividad térmica más que aceptables de 105 a $115 \mathrm{~W} / \mathrm{m} . \mathrm{k}$ y durezas del orden de los 30-33 HRC.

Por lo tanto, esta aleación es una alternativa de reemplazo importante a lo referente a aleaciones no ferrosas para moldes de inyección de plásticos o servicios de exigencia térmica/eléctrica.

\section{Agradecimientos}

Los autores a agradecen al Laboratorio de Investigaciones en Metalurgia Física, (LIMF) de la Facultad de Ingeniería de La Plata (UNLP), por facilitar el uso de sus instalaciones y equipamientos.

\section{REFERENCIAS}

1 Edens WW, Ingerson QF. Copper-nickel-silicon-chromium alloy having improved electrical conductivity, 1980, US patent $N^{\circ} 4191601$. Disponible en: http://www.uspto.gov/

2 Fischer RB, Harry JJ, Roach DB. Copper base Alloys, 1968, US patent № 2851353. Disponible en: http://www.uspto.gov/

3 Edens WW, Ingerson QF. Copper, Nickel, Silicon, Chromiun Alloys, 1991, US patent N 5028391. Disponible en: http://www.uspto.gov/

4 A Cohen. Heat Treating ASM Handbook, Vol. 4, American Society for Metals 1991.

5 Kerlins V. Fractography, ASM Handbook, Vol. 12, American Society for Metals 1987. 